\title{
Molecular dynamics simulations of mineral surface wettability in the presence of carbon dioxide and organics
}

\author{
EMILY WEI-HSIN SUN AND IAN BOURG
}

Princeton University

Presenting Author: esun@princeton.edu

Mineral surface wettability is an important property influencing multiphase flow in soils and sedimentary rocks. In particular, for $\mathrm{CO}_{2}$ abatement technologies that rely on trapping supercritical $\mathrm{CO}_{2}$ in sedimentary formations, the wettability of relevant mineral surfaces by water is a poorly constrained fundamental property influencing stratigraphic and residual trapping. Two prominent factors that may influence wetting observations are the presence of organics and scale. Theoretical studies have noted that adsorbed water films may hold a key to resolving many of the discrepancies in reported wettability data, but the transition from the droplet to the film is difficult to observe experimentally. This link between continuum and nanoscale observations can be elucidated using molecular dynamics (MD) and thermodynamic equations. We simulate water and $\mathrm{CO}_{2}$ at various pressures between quartz surfaces to probe the thickness of the adsorbed water film observed between the $\mathrm{CO}_{2}$ and quartz, and the radius of curvature of the fluid-fluid interface as a function of $\mathrm{CO}_{2}$ pressure. These results are discussed in the context of the relevant interfacial energies in Young's equation, and the Gibbs $\mathrm{CO}_{2}$ surface excesses at various interfaces. We show that the augmented Young-Laplace equation accurately captures the relationship between the observed radius of curvature, the capillary pressure between the bulk fluid phases, and the disjoining pressure in the adsorbed water film. We examine the thermodynamics of thin water films in novel depth and present a new methodology for characterizing curvature approaching a mineral interface and for comparing continuum and nanoscale manifestations of wettability. We find that discrepancies in both the experimantal and MD database may be influenced by proximity to solid surfaces and adsorbed wetting films. We further examine these behaviors by incorporating organic molecules into the system, changing the balance of interfacial tensions, and approximating a more realistic geologic carbon sequestration formation. 